\title{
The 6 year incidence of diabetes-associated autoantibodies in genetically at-risk children: the TEDDY study
}

\author{
Jeffrey P. Krischer • Kristian F. Lynch • Desmond A. Schatz • Jorma Ilonen • \\ Åke Lernmark • William A. Hagopian • Marian J. Rewers • Jin-Xiong She • \\ Olli G. Simell • Jorma Toppari • Anette-G. Ziegler • Beena Akolkar • \\ Ezio Bonifacio • the TEDDY Study Group
}

Received: 4 September 2014 / Accepted: 13 January 2015 / Published online: 10 February 2015

(C) Springer-Verlag Berlin Heidelberg 2015

\begin{abstract}
Aims/hypothesis Islet autoantibodies, in addition to elevated blood glucose, define type 1 diabetes. These autoantibodies are detectable for a variable period of time before diabetes
\end{abstract}

A complete list of the members of the TEDDY Study Group can be found in the electronic supplementary material (ESM).

Electronic supplementary material The online version of this article (doi:10.1007/s00125-015-3514-y) contains peer-reviewed but unedited supplementary material, which is available to authorised users.

\section{J. P. Krischer $(\bowtie) \cdot K$. F. Lynch}

Department of Pediatrics, Morsani College of Medicine, University

of South Florida, 3650 Spectrum Boulevard, Suite 100,

Tampa, FL 33612, USA

e-mail: jeffrey.krischer@epi.usf.edu

D. A. Schatz

Department of Pediatrics, College of Medicine, University of Florida, Gainesville, FL, USA

\section{J. Ilonen}

Department of Clinical Microbiology, University of Eastern Finland, Kuopio, Finland

J. Ilonen

Immunogenetics Laboratory, University of Turku, Turku, Finland

A. Lernmark

Department of Clinical Sciences, Skåne University Hospital SUS, Lund University/CRC, Malmo, Sweden

W. A. Hagopian

Pacific Northwest Diabetes Research Institute, Seattle, WA, USA

\section{J. Rewers}

Barbara Davis Center for Childhood Diabetes, University of

Colorado, Aurora, CO, USA

\section{J.-X. She}

Center for Biotechnology and Genomic Medicine, Medical College of Georgia, Georgia Regents University, Augusta, GA, USA onset. Thus, the occurrence of islet autoantibodies is associated with the beginning of the disease process. The age at, and order in, which autoantibodies appear may be associated with different genetic backgrounds or environmental exposures, or both.

O. G. Simell · J. Toppari

Department of Pediatrics, Turku University Hospital, Turku, Finland

J. Toppari

Department of Physiology, University of Turku, Turku, Finland

A.-G. Ziegler

Institute of Diabetes Research, Helmholtz Zentrum München,

Neuherberg, Germany

A.-G. Ziegler

Klinikum rechts der Isar, Technische Universität München,

München, Germany

\section{A.-G. Ziegler}

Forschergruppe Diabetes e.V., Helmholtz Zentrum München, Neuherberg, Germany

\section{B. Akolkar}

National Institute of Diabetes and Digestive and Kidney Diseases, Bethesda, MD, USA

\section{E. Bonifacio}

Center for Regenerative Therapies Dresden, Dresden University of Technology, Dresden, Germany 
Methods Infants with $H L A-D R$ high-risk genotypes (DR3/4, $D R 4 / 4, D R 4 / 8$ and $D R 3 / 3$ ) were enrolled and prospectively followed with standardised autoantibody assessments quarterly throughout the first 4 years of life and then semi-annually thereafter.

Results Autoantibodies appeared in 549/8,503 (6.5\%) children during 34,091 person-years of follow-up. Autoantibodies at $3(0.1 \%)$ and $6(0.2 \%)$ months of age were rare. Of the 549 , $43.7 \%$ had islet autoantibodies to insulin (IAA) only, 37.7\% had glutamic acid decarboxylase autoantibodies (GADA) only, $13.8 \%$ had both GADA and IAA only, $1.6 \%$ had insulinoma antigen-2 only and $3.1 \%$ had other combinations. The incidence of IAA only peaked within the first year of life and declined over the following 5 years, but GADA only increased until the second year and remained relatively constant. GADA only were more common than IAA only in $H L A-D R 3 / 3$ children but less common in HLA-DR4/8 children.

Conclusions/interpretation Islet autoantibodies can occur very early in life and the order of appearance was related to $H L A-D R-D Q$ genotype.

Keywords Autoimmunity - Diabetes in young children . $H L A-D R-D Q$ genotypes · Incidence · Islet autoantibodies . Type 1 diabetes

$\begin{array}{ll}\text { Abbreviations } \\ \text { FDR } & \text { First-degree relative } \\ \text { GADA } & \text { Glutamic acid decarboxylase autoantibodies } \\ \text { GP } & \text { General population } \\ \text { IA } & \text { Islet autoimmunity } \\ \text { IAA } & \text { Islet autoantibodies to insulin } \\ \text { IA-2A } & \text { Insulinoma antigen-2 } \\ \text { IQR } & \text { Interquartile range } \\ \text { PH } & \text { Proportional hazard } \\ \text { SNP } & \text { Single-nucleotide polymorphism } \\ \text { TEDDY } & \text { The Environmental Determinants of Diabetes in } \\ & \text { the Young }\end{array}$

\section{Introduction}

As an autoimmune disease, type 1 diabetes is defined by the presence of one or more known islet cell autoantibodies, in addition to elevated blood glucose above the threshold set by the American Diabetes Association [1]. It is also well established that these autoantibodies are detectable for some variable period of time before the clinical onset of diabetes. The number of detected autoantibodies is related to the risk of clinical onset, with the largest increase in risk associated with the presence of two or more autoantibodies $[2,3]$. Thus, it is natural to speculate that the initiation of the disease process begins with a single autoantibody followed by intermolecular epitope spreading to multiple autoantibodies, loss of insulin secretory capability resulting from a combination of beta cell destruction and inhibition of function, leading to metabolic changes, and finally diabetes.

The detection of islet autoantibodies in very young children has been reported to peak between 9 months and 2 years of age, with no seroconversion occurring at 3 or 6 months of age in children born to a mother or father with type 1 diabetes [3-5]. In a larger study of children with HLA-conferred genetic risk, the peak in the incidence of conversion to autoantibody positivity occurred at age 1-2 years with islet autoantibodies to insulin (IAA) appearing first most commonly [5]. In these studies, the sampling frequency affected the observed incidence rates and similar changes in the incidence of autoantibodies by the age they were seen. This paper reports the predominant subsets of the first appearance of IAA only, glutamic acid decarboxylase autoantibodies (GADA) only and insulinoma antigen-2A (IA-2A) only as well as any combination of the three in The Environmental Determinants of Diabetes in the Young (TEDDY) study, a large cohort of genetically at-risk individuals followed from birth with uniform sampling from 3 months of age onwards [6, 7]. We examined the temporal appearance of autoantibody subsets and the possible relationship with $H L A-D R-D Q$ genotype.

\section{Methods}

Participants The TEDDY study is a prospective cohort study funded by the National Institutes of Health and has the primary goal of identifying environmental causes of type 1 diabetes. It includes six clinical research centres - three in the USA (Colorado, Georgia/Florida, Washington) and three in Europe (Finland, Germany, Sweden). Detailed study design and methods have been previously published. For all study participants, written informed consents were obtained from a parent or primary carer, separately, for genetic screening and participation in the prospective follow-up. The high-risk genotypes for participants screened from the general population were as follows: $D R B 1 * 04-D Q A 1 * 03-D Q B 1 * 03: 02 / D R B 1 * 03-$ $D Q A 1 * 05-D Q B 1 * 02: 01$ (DR3/4), DRB1*04-DQA1*03$D Q B 1 * 03: 02 / D R B 1 * 04-D Q A 1 * 03-D Q B 1 * 03: 02(D R 4 / 4)$, $D R B 1 * 04-D Q A 1 * 03-D Q B 1 * 03: 02 / D R B 1 * 08-D Q A 1 * 04-$ $D Q B 1 * 04: 02(D R 4 / 8)$ and $D R B 1 * 03-D Q A 1 * 05$ $D Q B 1 * 02: 01 / D R B 1 * 03-D Q A 1 * 05-D Q B 1 * 02: 01$ (DR3/3). The $H L A-D R$ genotype will be used throughout as an abbreviation. The study was approved by local Institutional Review or Ethics Boards and is monitored by an External Evaluation Committee formed by the National Institutes of Health.

Non-HLA genotyping When the child was 9-12 months of age $(n=7,463)$, the HLA-DR-DQ genotypes were confirmed at the central HLA Reference Laboratory at Roche Molecular Systems, Oakland, CA, USA [8], together with three single- 
nucleotide polymorphism (SNP) primer pairs. These included the INS-23Hph1 (rs689), CTLA4 T17A (rs231775) and PTPN22 R620W (rs2476601). Briefly, the genomic DNA was extracted using Qiagen Kits (Qiagen, Hilden, Germany) and approximately $150 \mathrm{ng}$ DNA was used for PCR amplification. The polymorphic exon 2 of the $D R B 1, D Q B 1$ and $D Q A 1$ loci were specifically amplified by biotin-labelled primers. The DQA1 and DQB1 loci were co-amplified in a single reaction together with the three SNP primer pairs. Sequencespecific oligonucleotide probes were immobilised on a membrane in a linear fashion (strip). The DRB1 high-resolution strips contained 81 probes while the $D Q A 1 / D Q B 1+$ SNPs strips contained $15 D Q A 1$ and $40 D Q B 1$ probes, and two probes per SNP pair. The hybridisation of the amplicon and probe signal detection on the strip was semi-automated using a BeeBlot instrument (Bee Robotics, Caernarfon, Wales, UK). The genotype assignment was done by StripScan software developed by Roche (Oakland, CA, USA).

Islet autoantibodies IAA, GADA and IA-2A were measured in two laboratories by radiobinding assays [6, 7]. In the USA, all sera were assayed at the Barbara Davis Center for Childhood Diabetes at the University of Colorado, Denver; in Europe, all sera were assayed at the University of Bristol, UK. Both laboratories show high sensitivity and specificity as well as concordance [9]. All positive islet autoantibodies and $5 \%$ of negative samples were re-tested in the other reference laboratory and deemed confirmed if concordant. Persistent islet autoimmunity (IA) was defined as confirmed positive autoantibodies to insulin, GAD65 or IA-2 in at least two consecutive samples.

Maternal autoantibodies As children can be born with circulating maternal islet cell autoantibodies $[10,11]$ it was necessary to exclude positive results that were due to maternal IgG transmission when defining the child's autoantibody status. To distinguish between maternal autoantibodies and autoantibodies in the child, the status of the mother was measured when the child was aged 6 or 9 months. The child's autoantibody status was measured at 3 months of age and then every 3 months until 4 years of age. The child's autoantibody status was determined based on both maternal and child autoantibodies over the first 18 months of the study. If a maternal antibody was present, the child was not considered positive for that autoantibody unless either the child had a negative sample prior to their first positive sample or the autoantibody persisted beyond 18 months of age. If the mother was negative for autoantibodies, then all positive results were associated with the child. While important to distinguish true child autoantibodies from maternal autoantibodies, a limitation of this approach is that the child's positivity during the first 18 months of life could be masked by maternal autoantibodies.
Statistical methods Among the children who developed persistent confirmed islet autoantibodies, time to seroconversion was described as a median and interquartile range (IQR). Comparisons between groups of children who had different types of islet autoantibodies at seroconversion were performed using Mann-Whitney tests. Because of the different lengths of follow-up time in the study, the incidence of these diabetesrelated autoantibodies was described as a rate per 1,000 person-years. Exact $95 \%$ CIs in incidence rates were calculated using the $\chi^{2}$ relationship to the Poisson distribution [12]. Of interest was a comparison in the risks of different types of islet autoantibody seroconversion (i.e. GADA only at seroconversion, IAA only at seroconversion). Separate proportional hazards $(\mathrm{PH})$ models first examined factors related to the different types of islet autoantibody seroconversion (i.e. model 1 examined factors related to IAA only seroconversion, model $2 \mathrm{ex}$ amined factors related to GADA only seroconversion, etc.). The magnitudes of the associations were described by HRs with $95 \%$ CIs. Also, multiple logistic regression models compared the children with different types of islet autoantibody seroconversion directly to test for factors that distinguished between the different islet autoantibody seroconversion groups. The ORs describe the odds of a single autoantibody seroconversion being GADA only compared with IAA only.

Differences in islet autoantibody risk between a firstdegree relative (FDR) of an individual with type 1 diabetes as compared with children recruited from the general population (GP), HLA groups and countries were described first. As mentioned above, HLA-DR genotypes were generally used as abbreviations for the full $H L A-D R-D Q$ genotypes used in the study. Age was included in the multivariate logistic regression when comparing the different types of autoantibody seroconversion groups.

Unless stated otherwise, all analysis was pre-planned and multivariate analysis was performed where possible. A $p$ value $<0.05$ was considered significant. No correction for multiple comparisons was made. All $p$ values were two sided. SAS 9.3 (SAS Institute, Cary, NC, USA) was used for the statistical analyses and GraphPad PRISM 5.03 (GraphPad Software, San Diego, CA, USA) was used for graphs.

\section{Results}

Included in this analysis were 8,503 of the 8,676 children enrolled in TEDDY study (116 HLA ineligible and 57 with either no sample results or indeterminate autoantibody status were excluded). As of 30 April 2014, 549 children (6.5\%) had persistent confirmed autoantibodies during 34,091 personyears of follow-up until 6 years of age (16.1/1,000 personyears). Of the $549,43.7 \%$ (240) had IAA only, 37.7\% (207) GADA only, $13.8 \%$ (76) GADA and IAA, 1.6\% (9) IA-2A only and $3.1 \%(17)$ had other combinations. 


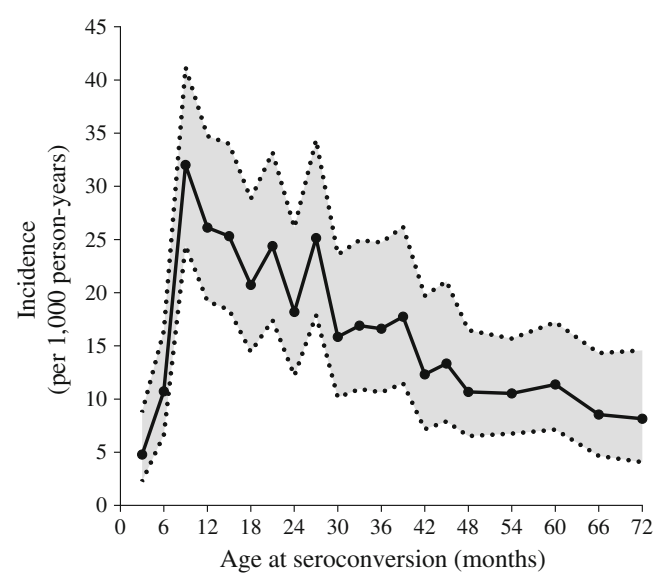

Fig. 1 Incidence of antibodies among 0- to 6-year-old children in the TEDDY study by age of seroconversion (incidence and $95 \%$ piecewise confidence bands). Autoantibodies appeared in 549/8,503 children

Ten children were autoantibody positive at 3 months of age and an additional 21 children developed autoantibodies at 6 months of age. The incidence of islet autoantibodies rose sharply until 9 months of age and declined slowly thereafter (Fig. 1). The incidence of IAA only was greatest within the first year of life and declined over the following 5 years; however, in contrast, the incidence of GADA only rose until the second or third year of life and remained relatively constant until 6 years of age (Fig. 2a). The incidence of simultaneous IAA and GADA was much less discernible and seemed to occur before 3 years of age (Fig. 2a, Table 1). Although the incidence overall was lower for GP than for FDR children, the incidence pattern by age was similar (Fig. 2b, c) and the relative disparity in the median time to seroconversion for IAA only (median 18 months) as compared with GADA only (median 33 months) was seen $(p<0.001$, Table 1$)$ in both the GP and the FDR (median 19 vs 28 months, $p<0.01$ ) populations.
The simultaneous appearance of IAA and GADA were intermediary between IAA only and GADA only (Table 1).

The delay in the incidence of GADA only, relative to IAA only, was evident in HLA-DR3/4 $(p<0.001)$ and $D R 4 / 8$ $(p<0.001)$ but not in the DR4/4 $(p=0.07)$ or $D R 3 / 3$ $(p=0.75)$ children (Fig. 3 ). The relative delay in the appearance of GADA only was statistically significant across all TEDDY study sites, with the exception of Germany (Table 1).

Overall, the appearance of GADA only relative to IAA only was close to even (odds of GADA only over IAA only was $207 / 240=0.86$ ). After accounting for age, FDRs, country and examining each of the four HLA genotypes separately, the adjusted ratio of GADA only relative to IAA only was significantly higher among the $H L A-D R 3 / 3$ children compared with other children (OR 4.5, 95\% CI 2.3, 8.8, $p<0.001$ ) and significantly lower among the $H L A-D R 4 / 8$ children (OR $0.44,95 \%$ CI $0.24,0.80, p=0.007)$.

The median (IQR) age of children having either IAA only or GADA only at seroconversion was 24 (13-40) months. After adjusting for HLA genotype, the age disparity favours IAA only under 13 months (OR $0.31,95 \%$ CI $0.17,0.57$, $p=0.0002$ ), IAA only from 13 to $<24$ months (OR 0.56 , $95 \% \mathrm{CI} 0.32,0.98, p=0.04$ ) and GADA only from 40 months of age onwards (OR 2.57, 95\% CI 1.44, 4.58, $p=0.001$ ), with the 24 to $<40$ month age group as the reference.

Non-HLA genotypes were next examined in relation to IAA only, GADA only and the ratio. The susceptible T allele of the PTPN22 SNP was associated with IAA only (HR 1.71, $95 \%$ CI 1.36, 2.16, $p<0.001$ ) and GADA only (HR 1.51, 95\% CI 1.16, 1.99, $p=0.003$ ) but not with the GADA/IAA ratio. The susceptible A allele of the INS SNP was associated with IAA only (HR 1.85, 95\% CI 1.44, 2.38, $p<0.001$ ) and the minor $\mathrm{G}$ allele of the CTLA4 SNP with risk of GADA only (HR 1.28, 95\% CI 1.05, 1.55, $p=0.02$ ) and both were associated a

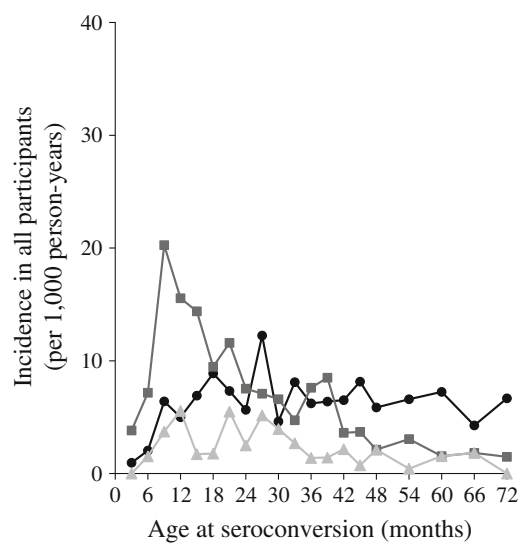

b

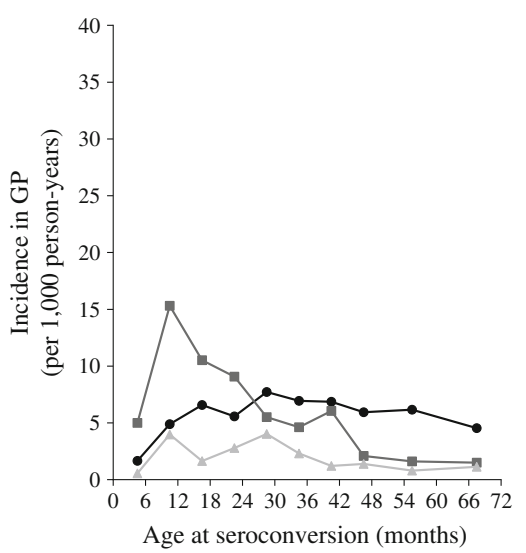

c

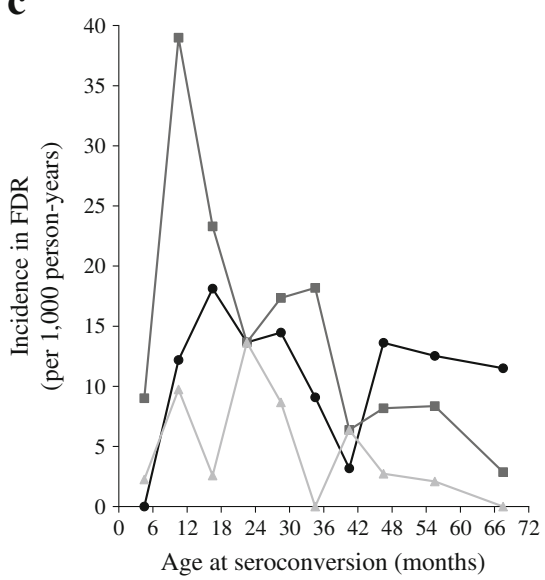

Fig. 2 Incidence of IAA only (dark grey lines and squares), GADA only (black lines and circles) and IAA and GADA only (light grey lines and triangles) in all participants (a), GP (b) or FDR (c) of a proband with type 1 diabetes. In (a), of 8,503 children, IAA only appeared in 240, GADA only in 207 and IAA and GADA in 76. In (b), of 7,584 children, IAA only appeared in 184, GADA only in 166 and IAA and GADA in 58. In (c), of 919 children, IAA only appeared in 56, GADA only in 41 and IAA and GADA in 18 


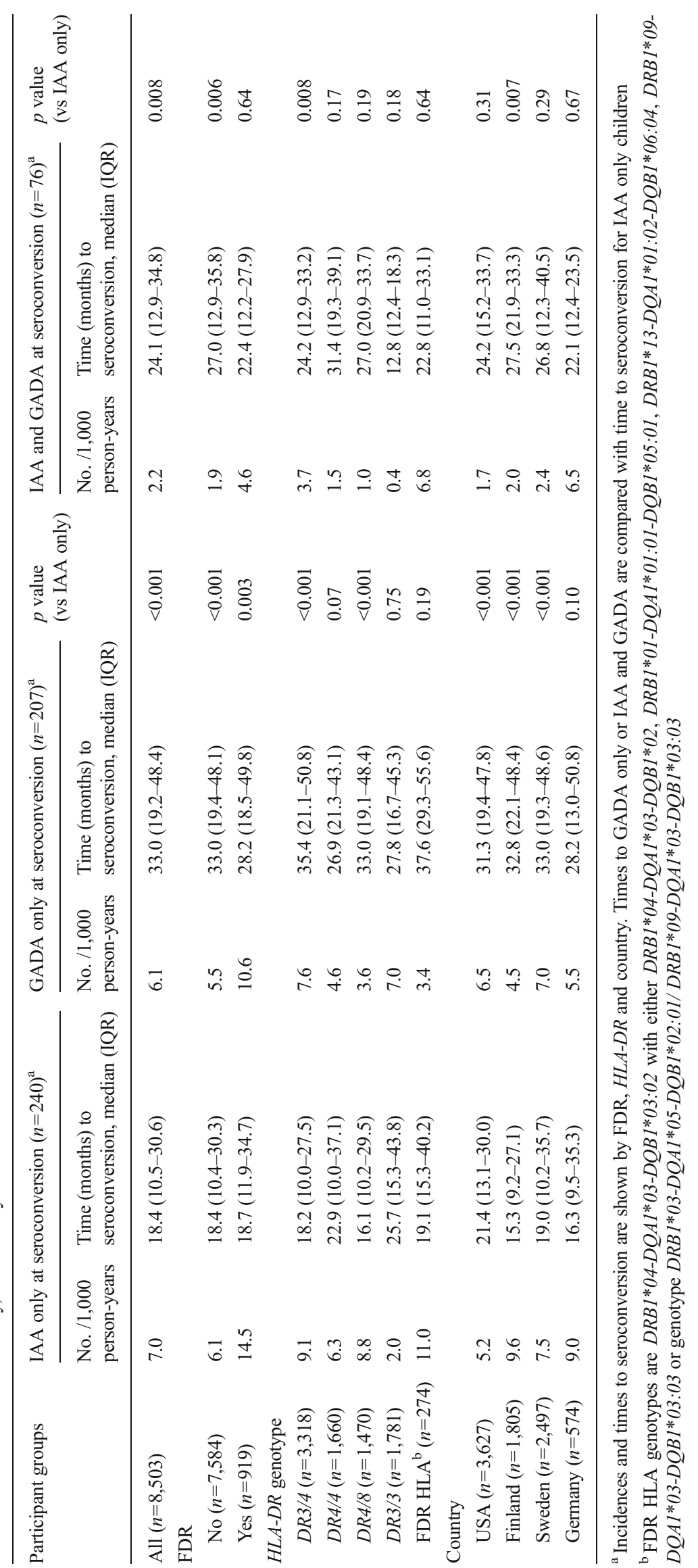


with the GADA/IAA ratio (INS OR $0.55,95 \%$ CI $0.39,0.82$, $p=0.003$ and CTLA4 OR 1.40,95\% CI 1.02, 1.92, $p=0.04$ ).

Finally, the presence of maternal autoantibodies and the sex of the child were examined separately in relation to the risk of the child's autoantibodies. The presence of maternal autoantibodies showed a tendency towards an association on risk for IAA only (HR $0.49,95 \%$ CI $0.22,1.06, p=0.07$ ) and GADA only (HR $0.40,95 \%$ CI $0.14,1.10, p=0.07$ ) but neither was statistically significant. The risk of IAA was lower in girls than in boys (HR 0.69, 95\% CI 0.53, 0.89, $p=0.005$ ) but no association was seen with risk of GADA only (HR $0.90,95 \% \mathrm{CI}$ $0.69,1.19, p=0.47$ ) or the GADA/IAA ratio (OR $1.29,95 \%$ CI $0.83,2.00, p=0.26$ ).

While some variables may increase or decrease the risk of an autoantibody appearing alone, they may not alter the relative incidence of the autoantibodies. After adjusting for age of seroconversion, $H L A-D R-D Q$, FDR and country, GADA only (compared with IAA only) at the time of seroconversion was protective for multiple autoantibodies in the first year after seroconversion (HR 0.60, 95\% CI 0.43, 0.85, $p=0.004$ ) but thereafter the HR changed significantly $(p<0.01)$ and protection was no longer seen (HR 1.24, 95\% CI 0.73, 2.11, $p=0.43$ ).

Although not statistically significant, a similar early association was seen for the risk of type 1 diabetes. Participants who had GADA only were marginally less likely to develop type 1 diabetes in the first year after seroconversion compared with those who had IAA only (HR $0.35,95 \%$ CI 0.12, 1.04, $p=0.06)$. The protective effect did reach statistical significance in the first 2 years after seroconversion as compared with participants who had IAA only (HR $0.47,95 \%$ CI 0.23 , $0.94, p=0.03)$. This protection was no longer seen after the second year (HR 0.92, 95\% CI 0.51, 1.67, $p=0.78$ ).

\section{Discussion}

TEDDY is perhaps the largest study to date to screen and enroll the highest genetically at-risk infants from families in the GP $(89 \%)$ and from families with an FDR with type 1 diabetes (11\%). These proportions reflect epidemiological data indicating that only $13-15 \%$ of children with newly diagnosed type 1 diabetes have an FDR with the disease [13, 14].

The size of the TEDDY study cohort and the standardised approach to autoantibody assessment [9] give rise to a high level of precision when estimating incidence and associated risk factors. Diabetes-related autoantibodies that are not maternal in origin may be found possibly as early as 3 months of age and with increasing numbers in children at 6 months of age, suggesting a narrow window of possible exposure that may affect the initiation of IA. Similar observations were
Fig. 3 Incidence of IAA only (dark grey lines and squares), GADA only (black lines and circles) and IAA and GADA only (light grey lines and triangles) by HLA genotype: $D R 3 / 4$ (a), $D R 4 / 8$ (b), $D R 4 / 4$ (c) and $D R 3 / 3$ (d). In (a), of 3,318 children, IAA only appeared in 120, GADA only in 101 and IAA and GADA in 49. In (b), of 1,470 children, IAA only appeared in 51, GADA only in 21 and IAA and GADA in 6. In (c), of 1,660 children, IAA only appeared in 42, GADA only in 31 and IAA and GADA in 10. In (d), of 1,781 children, IAA only appeared in 14 children, GADA only in 50 and IAA and GADA in 3
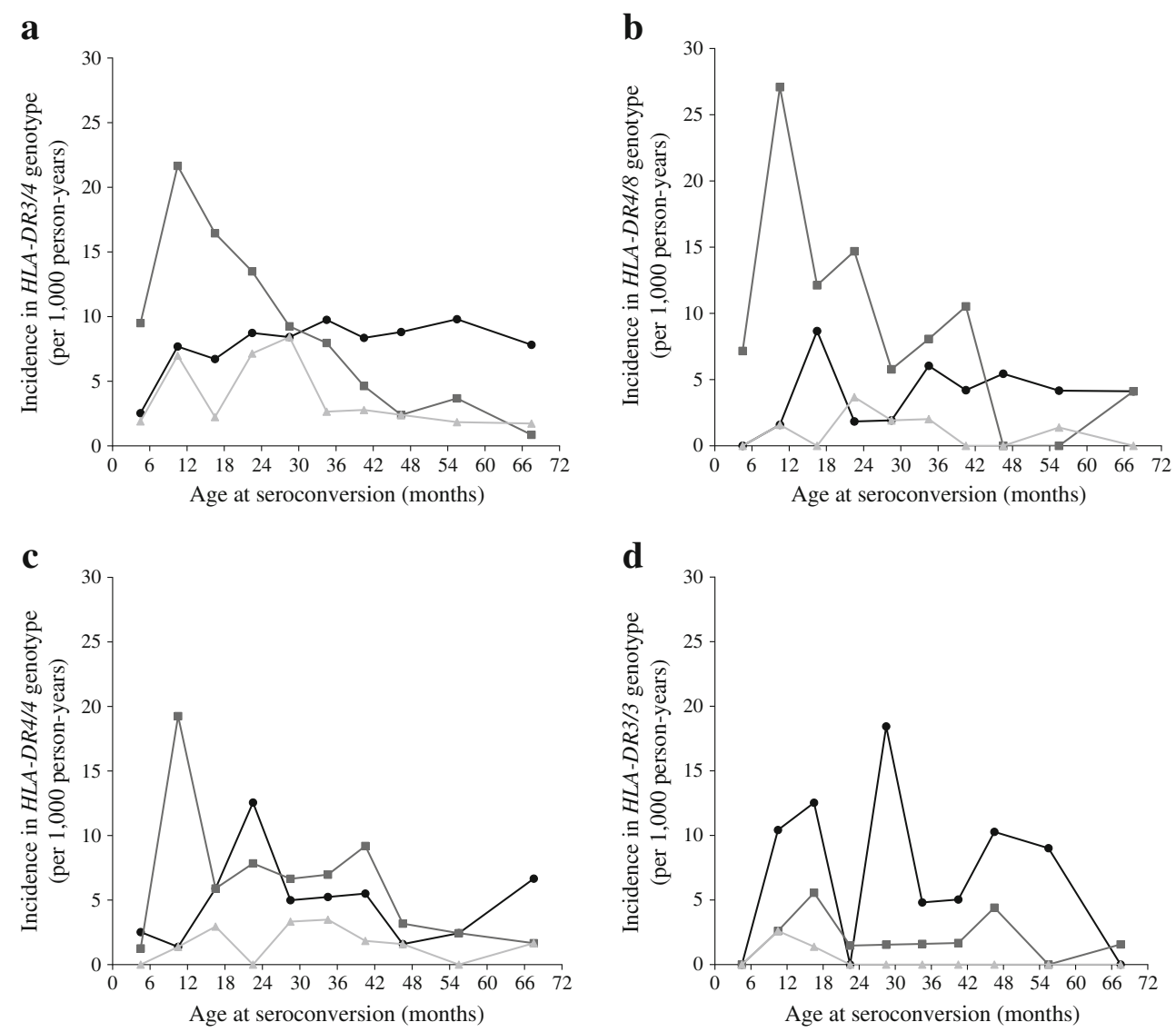
reported in the DiPP (Diabetes Prediction and Prevention) [5] and BABY DIAB (Baby Diabetes) [4] studies. Our observation that a higher percentage of children seroconverting at 6 months of age had the HLA-DR3/4 genotype $(81.0 \%)$ compared with children seroconverting at 3 months $(50.0 \%)$ or seroconverting from 9 months onwards $(49.6 \%)$ (test of difference in percentage across the three groups; $p=0.02$ ) suggests an important genetic interaction. Our observations that the appearance of IAA only tends to be associated with the $H L A-D R 4$ haplotype while GADA only was associated with the HLA-DR3 haplotype is of considerable interest. Similar associations between HLA and islet autoantibodies have previously been observed at the time of clinical diagnosis $[15,16]$ extended to the observation that IA-2A was associated with $H L A-D R 4$ but negatively associated with $H L A-D R 3[15,17]$. The strong association between HLA and the seroconversion to a specific islet autoantibody therefore underscores prior observations that the association between HLA and type 1 diabetes at the time of clinical diagnosis are secondary to a primary association between HLA and an autoimmune response to either IAA only or GADA only. Further studies are needed to clarify whether the association between HLA and the seroconversion to IAA only or GADA only in turn as secondary to a primary association with a hypothetical trigger.

The differences seen between $H L A-D R$ genotypes and between the effects of INS and CTLA4 gene polymorphisms also suggest specific interactions or immunogenic pathways (e.g. the total absence of $D R 3 / 3$ and a higher proportion of $D R 3 / 4$ among those who seroconverted at 3 or 6 months as compared with those who seroconverted at 9 months of age or after). Although genes in the HLA region remain the most important genetic risk factors for type 1 diabetes, other non-HLA genetic factors may contribute to seroconversion to IAA, GADA or both. The TEDDY study recently revealed that the non-HLA genes PTPN22, ERBB3, SH2B3 and INS were primarily associated with seroconversion to IA, the first step in the pathogenesis of type 1 diabetes, and the progression of the disease [18]. TEDDY has carefully documented possible exposures that may be important to IA including prenatal and perinatal events, the introduction of first foods, breast-feeding practices, early childhood illness, changes in the gut microbiome and gene expression in blood cells, as well as vaccinations and immunisations [3]. The advantage of delineating different subgroups from the larger TEDDY cohort is that they may be more homogeneous with respect to genetic risk factors and environmental exposures and this may facilitate the discovery of triggers from a relatively age-restricted set of possibilities. The present TEDDY data suggest for the first time that triggers of IAA only may differ from those of GADA only and that islet autoimmunity may be the result of a combination of triggers. It cannot be excluded that the mechanisms by which a trigger induces IA are associated with $H L A-D R$ or $H L A-D Q$, or both, haplotypes. The findings are consistent in both FDRs and the GP.

\section{Conclusions}

Diabetes-related persistent confirmed islet autoantibodies first appeared singly with IAA only appearing at an earlier age than GADA only. Autoantibodies may occur as early as 3 months of age. The order of appearance was related to $H L A-D R-D Q$ genotypes and to that extent carry differential risks for type 1 diabetes. Also, the appearance of multiple autoantibodies following GADA only antibodies was slower than following the appearance of IAA only. The age difference in the relative incidence of GADA and IAA was quite dramatic, consistent with the generally held view that IAA appears first. The appearance of IAA would mark only a fraction of the very young as the incidence dropped precipitously. However, the simultaneous appearance of IAA and GADA suggest an intermediary phenotype with marked increased risk for type 1 diabetes (Figs 2,3). The TEDDY study identified ten children who had persistent confirmed islet autoantibodies from 3 months of age and an additional 21 who had them by 6 months of age. These children may point to different risk factors, limited to genetics, and prenatal or perinatal exposures, which may suggest a different aetiological pathway for IA and type 1 diabetes than exposures occurring later in life. Although this is a very large study of children with increased genetic risk from the GP or who are FDRs of an individual with type 1 diabetes, the ongoing follow-up should prove useful to fully describe the relationship between the order of autoantibody appearance and the risk of type 1 diabetes.

Acknowledgements A special acknowledgement goes to the TEDDY families for their continued participation in this wonderful study.

Funding This work was funded by U01 DK63829, U01 DK63861, U01 DK63821, U01 DK63865, U01 DK63863, U01 DK63836, U01 DK63790, UC4 DK63829, UC4 DK63861, UC4 DK63821, UC4 DK63865, UC4 DK63863, UC4 DK63836, and UC4 DK95300 and Contract No. HHSN267200700014C from the National Institute of Diabetes and Digestive and Kidney Diseases (NIDDK), National Institute of Allergy and Infectious Diseases (NIAID), National Institute of Child Health and Human Development (NICHD), National Institute of Environmental Health Sciences (NIEHS), Juvenile Diabetes Research Foundation (JDRF), and Centers for Disease Control and Prevention (CDC). This work supported in part by the NIH/NCATS Clinical and Translational Science Awards to the University of Florida (UL1 TR000064) and the University of Colorado (UL1 TR001082).

Duality of interest The authors declare that there is no duality of interest associated with this manuscript.

Contribution statement All authors attest to meeting ICMJE uniform requirements for authorship by making substantial contributions to the conception and design of this study, acquisition, analysis and interpretation of the data and drafting or revising the article for intellectual content. 
All have given final approval of the published version. JPK is responsible for the integrity of the work as a whole.

\section{References}

1. (1997) Report of the expert committee on the diagnosis and classification of diabetes mellitus. Diabetes Care 20:1183-1197

2. Orban T, Sosenko JM, Cuthbertson D et al (2009) Pancreatic islet autoantibodies as predictors of type 1 diabetes in the diabetes prevention trial-type 1. Diabetes Care 32:2269-2274

3. Ziegler AG, Rewers M, Simell O et al (2013) Seroconversion to multiple islet autoantibodies and risk of progression to diabetes in children. JAMA 309:2473-2479

4. Ziegler AG, Bonifacio E (2012) Age-related islet autoantibody incidence in offspring of patients with type 1 diabetes. Diabetologia 55:1937-1943

5. Ilonen J, Hammais A, Laine AP et al (2013) Patterns of beta-cell autoantibody appearance and genetic associations during the first years of life. Diabetes 62:3636-3640

6. TEDDY Study Group (2007) The Environmental Determinants of Diabetes in the Young (TEDDY) study: study design. Pediatr Diabetes 8:286-298

7. TEDDY Study Group (2008) The Environmental Determinants of Diabetes in the Young (TEDDY) Study. Ann N Y Acad Sci 1150:1-13

8. Hagopian WA, Erlich H, Lernmark A et al (2011) The Environmental Determinants of Diabetes in the Young (TEDDY): genetic criteria and international diabetes risk screening of 421000 infants. Pediatr Diabetes 12:733-743

9. Bonifacio E, Yu L, Williams AK et al (2010) Harmonization of glutamic acid decarboxylase and islet antigen-2 autoantibody assays for national institute of diabetes and digestive and kidney diseases consortia. J Clin Endocrinol Metab 95:3360-3367

10. Koczwara K, Bonifacio E, Ziegler AG (2004) Transmission of maternal islet antibodies and risk of autoimmune diabetes in offspring of mothers with type 1 diabetes. Diabetes 53:1-4

11. Lynch KF, Lernmark B, Merlo J, Cilio CM, Ivarsson SA, Lernmark A (2008) Cord blood islet autoantibodies and seasonal association with the type 1 diabetes high-risk genotype. J Perinatol 28:211-217

12. Ulm K (1990) A simple method to calculate the confidence interval of a standardized mortality ratio (SMR). Am J Epidemiol 131:373375

13. O'Leary LA, Dorman JS, LaPorte RE et al (1991) Familial and sporadic insulin-dependent diabetes: evidence for heterogeneous etiologies? Diabetes Res Clin Pract 14:183-190

14. Dahlquist G, Blom L, Holmgren G et al (1985) The epidemiology of diabetes in Swedish children 0-14 years - a six-year prospective study. Diabetologia 28:802-808

15. Graham J, Hagopian WA, Kockum I et al (2002) Genetic effects on age-dependent onset and islet cell autoantibody markers in type 1 diabetes. Diabetes 51:1346-1355

16. Mäkinen A, Härkönen T, Ilonen J, Knip M (2008) Characterization of the humoral immune response to islet antigen 2 in children with newly diagnosed type 1 diabetes. Eur J Endocrinol 159:19-26

17. Vermeulen I, Weets I, Asanghanwa M et al (2011) Contribution of antibodies against IA-2beta and zinc transporter 8 to classification of diabetes diagnosed under 40 years of age. Diabetes Care 34:17601765

18. Torn C, Hadley D, Lee HS et al (2014) Role of type 1 diabetes associated SNPs on risk of autoantibody positivity in the TEDDY Study. Diabetes. doi:10.2337/db14-1497 